\title{
Uretroplastía con injerto de mucosa oral en pacientes con estenosis uretral anterior. Estudio comparativo con tratamiento convencional
}

\author{
Uretroplasty with oral mucosal graft in patients with anterior urethral stenosis. \\ Comparative study with conventional treatment
}

\author{
Armando Alonso-Morales ${ }^{1,2}$, Álvaro J. Montiel-Jarquín ${ }^{1 *}$, Carlos A. Camarillo-Noriega1, \\ José A. Parra-Salazar', Giovanni A. Romero-Tlalpan,2, Arturo García-Galicia ${ }^{1}$ y \\ Jorge Loría-Castellanos ${ }^{3}$ \\ 'Dirección de Educación e Investigación en Salud, Unidad Médica de Alta Especialidad, Hospital de Especialidades de Puebla, Instituto Mexicano \\ del Seguro Social (IMSS), Puebla, Puebla; ${ }^{2}$ Escuela de Medicina, Universidad Popular Autónoma del Estado de Puebla, Puebla Puebla; ${ }^{3}$ División \\ de Proyectos Especiales en Salud, IMSS, Ciudad de México. México
}

\begin{abstract}
Resumen
Antecedentes: El manejo con uretroplastía de la estenosis uretral es una opción quirúrgica fundamental, y comparar la uretroplastía con injerto de mucosa oral con la uretroplastía término-terminal tiene trascendencia para conocer la diferencia entre ambas técnicas. Objetivo: Comparar la uretroplastía con injerto de mucosa oral con la uretroplastía término-terminal en pacientes con estenosis uretral tipo Jordan C, D y E. Método: Cuasiexperimento realizado en pacientes con estenosis uretral anterior operados de uretroplastía con anastomosis término-terminal o con injerto de mucosa bucal. Resultados: Veintinueve pacientes con una media de edad de 50.7 años, 6 diabéticos, 9 hipertensos, longitud media de la estenosis de $3.6 \mathrm{~cm}, 19$ operados con uretroplastía termino-terminal (grupo 1) y uretroplastía con mucosa bucal (grupo 2). El Índice Internacional de Síntomas Prostáticos promedio preoperatorio fue grave en la mayoría de los pacientes (93\%). Hubo mejoría significativa en el posoperatorio en ambos grupos ( $p$ $=0.0001$ y $p=0.0011$ ), así como en los resultados uroflujométricos $(p=0.0046$ y $p=0.00062)$. Conclusiones: Ambos procedimientos lograron mejorías significativas en la sintomatología urinaria a los 6 meses en comparación con los valores preoperatorios.
\end{abstract}

Palabras clave: Clasificación Clavien-Dindo. International prostate symptoms score. Uretroplastía con injerto de mucosa bucal. Uretroplastía término-terminal. Uroflujometría.

\begin{abstract}
Background: Urethroplasty management of urethral stricture is a fundamental surgical option, and comparing urethroplasty with oral mucosal graft with end-to-end urethroplasty is important to know the difference between both techniques. Objective: To compare urethroplasty with oral mucosal graft with end-to-end urethroplasty in patients with urethral stricture type Jordan $C, D$ and E. Method: Quasi-experiment performed in patients with anterior urethral stricture operated with urethroplasty with end-to-end anastomosis or with oral mucosal graft. Results: Twenty-nine patients with a mean age of 50.7 years, 6 diabetic, 9 hypertensive, mean stenosis length of $3.6 \mathrm{~cm}, 10$ with end-to-end urethroplasty (group 1) and 19 operated with urethroplasty with buccal mucosa (group 2). The average preoperative International Prostatic Symptom Index was severe in most

Correspondencia:

*Álvaro J. Montiel-Jarquín

Norte, 2004

Col. Centro

Fecha de recepción: 20-02-2020

C.P. 72000 , Puebla, Pue., México

E-mail: alvaro.montielj@imss.gob.mx; dralmoja@ hotmail.com

Fecha de aceptación: 31-05-2020

DOI: $10.24875 / \mathrm{CIRU} .20000123$ (http://creativecommons.org/licenses/by-nc-nd/4.0/).
\end{abstract}


patients (93\%). There was significant improvement postoperatively in both groups ( $p=0.0001$ and $p=0.0011)$, as well as in uroflowmetric results $(p=0.0046$ and $p=0.00062)$. Conclusions: Both procedures achieved significant improvements in urinary symptomatology at 6 months compared to preoperative values.

Key words: Clavien-Dindo classification. International Prostate Symptoms Score. Oral mucosa graft urethroplasty. End to end urethroplasty. Uroflowmetry.

\section{Introducción}

La estenosis uretral es una disminución de la luz de la uretra. La estenosis uretral anterior va desde el inicio de la porción bulbar hasta la porción peneana y es una enfermedad en la cual el cuerpo esponjoso se encuentra debajo del epitelio uretral, lo que lleva a un proceso cicatricial que se extiende a través del tejido del cuerpo esponjoso y de las estructuras adyacentes. Puede permanecer asintomática durante cierto tiempo, aunque posteriormente se pueden presentar síntomas miccionales importantes'.

La mayoría de las veces es traumática y con frecuencia pasa inadvertida hasta que el paciente experimenta síntomas miccionales ${ }^{2}$. Las lesiones uretrales posteriores se consideran traumáticas por definición y producen defectos obliterativos o casi obliterativos asociados a fibrosis extensa interpuesta entre los extremos lesionados de la uretra ${ }^{3}$.

En general, los pacientes presentan síntomas miccionales como pujo y tenesmo, y síntomas obstructivos como goteo posmiccional, disminución del chorro urinario, alargamiento del tiempo de micción, infecciones recurrentes del tracto urinario (epididimitis y prostatitis) y en algunos casos retención urinaria ${ }^{4,5}$.

La localización y la profundidad pueden establecerse con estudios radiográficos, ultrasonografía o uretrocistoscopía. La profundidad y la densidad de la cicatriz se determinan mediante exploración física, y el aspecto de la uretra mediante estudios con contraste y evaluación ecográfica ${ }^{6}$. La uretrografía retrograda y la cistouretrografía miccional son los estudios radiológicos diagnósticos de elección para estudiar estrecheces uretrales ${ }^{7}$. La clasificación de la estenosis uretral, de acuerdo con Jordan (1987), es en seis grados: A) pliegue mucoso; B) en iris; C) espesor completo con mínima espongiofibrosis; D) espongiofibrosis de espesor completo; E) inflamación y fibrosis que afecta tejidos fuera del cuerpo esponjoso; y F) estenosis compleja complicada con fístula ${ }^{8}$.

La sintomatología es variada y puede incluir tenesmo, poliuria, incontinencia urinaria, disminución del chorro urinario, pujo y nicturia. Los síntomas se evalúan con el Índice Internacional de Síntomas Prostáticos (IPSS, International Prostate Symptoms Score), el cual brinda información para estadificarlos en leves, moderados y graves ${ }^{8}$.

El tratamiento puede ser médico o quirúrgico, dependiendo del grado de obstrucción. El quirúrgico se reserva para los grados C a F y depende de la longitud, el sitio y la gravedad de la obstrucción. Si la longitud de la estenosis es corta y el estrechamiento luminal no es grave, los procedimientos pueden ser menos invasivos, como la dilatación uretral, la uretrotomía interna o la implantación temporal de un stent ${ }^{9}$. Las estenosis en la uretra bulbar que miden menos de $1.5 \mathrm{~cm}$ y que no están asociadas con espongiofibrosis densa y profunda (lesiones en silla de montar) pueden tratarse con una uretrotomía interna, con tasas de éxito de hasta el $74 \%{ }^{10}$.

La uretroplastía abierta ofrece diversos resultados. En estenosis de segmentos mayores de $2 \mathrm{~cm}$ ocasionadas por instrumentaciones urológicas se prefiere la uretroplastía con injerto de mucosa, y en estenosis de $2 \mathrm{~cm}$ o menos ocasionadas por trauma uretral secundario a lesiones por accidente automovilístico con fractura de pelvis se prefiere la anastomosis término-terminal ${ }^{1,3}$. Sin embargo, se debe tomar en cuenta la experiencia del urólogo en la realización de cada una de estas técnicas. Debido a que en la literatura hay poca evidencia de la superioridad de una técnica con respecto a las otras, se decidió realizar este estudio cuyo objetivo es comparar los resultados de la uretroplastía con injerto de mucosa bucal con los de la uretroplastía término-terminal en pacientes con estenosis uretral anterior mayor de $1.5 \mathrm{~cm}$ y grados C, D y E de la clasificación de Jordan.

\section{Método}

Estudio observacional, cuasiexperimental, realizado en pacientes con estenosis uretral anterior, de grados C, D y E de la clasificación de Jordan, a quienes se realizó uretroplastía de enero de 2016 a diciembre de 2018. 
Tabla 1. Valores iniciales de la longitud de la estenosis, el IPSS y la uroflujometría por grupo de pacientes, y sus diferencias (prueba de Levene)

\begin{tabular}{|c|c|c|c|c|c|}
\hline Tipo de cirugía & & $\mathbf{N}$ & Media & DE & $\mathrm{p}$ \\
\hline \multirow[t]{2}{*}{ Longitud de la estenosis (cm) } & Anastomosis término-terminal & 10 & 3.80 & 1.989 & 0.91 \\
\hline & Uretroplastía con injerto de mucosa oral & 19 & 3.58 & 1.953 & \\
\hline \multirow[t]{2}{*}{ IPSS } & Anastomosis término-terminal & 10 & 21.60 & 3.658 & 0.27 \\
\hline & Uretroplastía con injerto de mucosa oral & 19 & 23.58 & 2.479 & \\
\hline \multirow[t]{2}{*}{ Uroflujometría } & Anastomosis término-terminal & 10 & 10.10 & 0.568 & 0.13 \\
\hline & Uretroplastía con injerto de mucosa oral & 19 & 10.37 & 0.831 & \\
\hline
\end{tabular}

DE: desviación estándar; IPSS: Escala Internacional de Síntomas Prostáticos.

Se incluyeron hombres operados mediante uretroplastía término-terminal (grupo 1) o con injerto de mucosa bucal (grupo 2), mayores de 18 años, sin infección de vías urinarias previa a la cirugía, con estenosis de origen traumático y de grados $\mathrm{C}, \mathrm{D}$ y $\mathrm{E}$ de la clasificación de Jordan. Se excluyeron los pacientes con hipospadias o epispadias, y se eliminaron aquellos que fallecieron o que solicitaron salir del estudio.

Se analizaron las variables siguientes: tipo de uretroplastía, grado de estenosis uretral, longitud de la estenosis medida por uretrografía, tipo de trauma que ocasionó la estenosis, enfermedades concomitantes presentadas por los pacientes, complicaciones posquirúrgicas de acuerdo con la escala de Clavien-Dindo, síntomas prequirúrgicos y posquirúrgicos según el IPSS, y realización de uretrotomía interna óptica (UTIO) previa a la cirugía. Las evaluaciones se realizaron al inicio y a los 6 meses de la operación. Se utilizó estadística descriptiva, con medidas de tendencia central y de dispersión. Para la comparación entre variables de interés se emplearon las pruebas de ji al cuadrado, $U$ de Mann Whitney y $D$ de Cohen para ver el tamaño del efecto, y exacta de Fisher dependiendo del tipo y de la distribución de las variables, con el programa SPSS v.25 para Windows.

El protocolo fue debidamente registrado y aprobado por el comité de ética en investigación médica de la unidad participante, y en todo momento se preservó el anonimato de los pacientes.

\section{Resultados}

En total se incluyeron 30 pacientes, de los cuales uno fue eliminado por cursar con hipospadias. De los 29 pacientes, 10 (34\%) fueron operados con uretroplastía y anastomosis término-terminal (grupo 1) y $19(66 \%)$ con uretroplastía e injerto de mucosa bucal (grupo 2).

Los valores preoperatorios de la longitud de la estenosis, del IPSS y de la uroflujometría por grupo de pacientes, así como la diferencia entre grupos, se muestran en la tabla 1.

La edad media de los pacientes fue de $51.6 \pm 15.8$ años (rango: 25-86). Los valores medios preoperatorios de creatinina, glucosa, IPSS y uroflujometría fueron $0.86,102.1,22.9$ y 10.28, respectivamente; los valores postoperatorios de estos mismos parámetros fueron $0.95,101.4,12.52$, y 13.41 , respectivamente (Tabla 2).

La localización más frecuente de la estenosis fue la uretra bulbar, con $21(72.4 \%)$ casos, seguida de la uretra peneana con $5(17.2 \%)$ y de ambas porciones uretrales con $3(10.3 \%)$. La longitud media de la estenosis uretral en el grupo 1 fue de $4 \pm 2.16 \mathrm{~cm}$ (rango: 2-8) y en el grupo 2 fue de $3.68 \pm 2.12$ (rango: 2-8) La etiología de la estenosis fue traumática en todos los casos.

La comorbilidad presentada fue la siguiente: en el grupo 1, diabetes mellitus tipo 2 en 4 pacientes e hipertensión arterial sistémica en 5 pacientes; en el grupo 2, diabetes mellitus tipo 2 en 2 pacientes e hipertensión arterial sistémica en 4 pacientes. Se realizó UTIO a cinco pacientes del grupo 1 y a 13 pacientes del grupo 2.

Las complicaciones posquirúrgicas se dividieron en tres tipos de acuerdo con la clasificación de Clavien-Dindo: complicaciones mayores en $5(17.2 \%)$ pacientes, complicaciones menores en 7 (24.1\%) y sin complicaciones en 17 (58.6\%) pacientes. La asociación entre las 
Tabla 2. Valores de creatinina, glucemia, IPSS y uroflujometría en los pacientes incluidos en el estudio

\begin{tabular}{lccccc}
\hline & $\mathbf{n}$ & Mínimo & Máximo & Media & DE \\
\hline Creatinina preoperatoria & 29 & 0.65 & 1.20 & 0.86 & 0.13 \\
Creatinina posoperatoria & 29 & 0.62 & 1.56 & 0.95 & 0.14 \\
Glucemia preoperatoria & 29 & 75 & 160 & 102.10 & 20.47 \\
Glucemia posoperatoria & 29 & 84 & 157 & 101.41 & 20.44 \\
IPSS preoperatorio & 29 & 15 & 30 & 22.90 & 3.08 \\
IPSS posoperatorio & 29 & 5 & 36 & 12.52 & 8.35 \\
Uroflujometría preoperatoria & 29 & 9 & 12 & 10.28 & 0.75 \\
Uroflujometría posoperatoria & 29 & 7 & 18 & 13.41 & 2.65 \\
\hline DE: desviación estándar; IPSS: Escala Internacional de Síntomas Prostáticos.
\end{tabular}

complicaciones posquirúrgicas y el procedimiento realizado se muestra en la tabla 3 .

La sintomatología posoperatoria de acuerdo con los resultados del IPSS fue leve en $13(44.8 \%)$ pacientes, moderada en $12(41.4 \%)$ y grave en $4(13.8 \%)$. En la tabla 4 se detalla la presentación de síntomas por grupo de pacientes.

En la tabla 5 se presenta la comparación entre los valores basal y final del IPSS, así como de la uroflujometría, en ambos grupos de pacientes, y se ve que hay diferencia entre las intervenciones basal y final de la cirugía.

La prueba $D$ de Cohen para evaluar el efecto mostró una diferencia entre grupos de 0.37 para el IPSS y de 0.64 para la uroflujometría.

\section{Discusión}

La estenosis uretral anterior ocasiona sintomatología urinaria baja. Sus complicaciones pueden llegar a ser graves y van desde infecciones hasta procesos obstructivos. La uretroplastía en sus modalidades puede resolver estas complicaciones.

En esta serie, en la que se evalúa la uretroplastía con injerto de mucosa bucal comparándola con la uretroplastía término-terminal como tratamiento convencional, se encontró que la uretroplastía con injerto de mucosa bucal ofrece resultados equiparables, con una tasa de éxito global del $82.8 \%$ y una tasa de fracaso del $17.2 \%$, con una tasa de fracaso menor que la de la uretroplastía término-terminal (10.5\% contra 30\%).

Los pacientes sometidos a UTIO antes de la uretroplastía fueron el $62.1 \%$ del grupo 1 y el $68.42 \%$ del grupo 2, dato similar a lo reportado en la literatura ${ }^{10,11}$, debido a que la UTIO se consideraba como tratamiento para la estenosis uretral. Actualmente, las recomendaciones de los expertos en el manejo de esta patología recomiendan no realizar UTIO en estenosis mayores de $1 \mathrm{~cm}$ y grados C, D y E de la clasificación Jordan, pero en esta serie sí se realizón ${ }^{11-13}$.

El lugar anatómico más frecuente de la estenosis fue la uretra bulbar $(72.4 \%)$, en segundo lugar la uretra peneana (17.2\%) y por último ambas porciones de la uretra $(10.3 \%)$, de modo similar a lo reportado en la literatura ${ }^{9,14,15}$.

En cuanto a la longitud media de la estenosis uretral, fue de $4.2 \mathrm{~cm}$. Las estenosis mayores de $8 \mathrm{~cm}$ se manejaron con mucosa bucal en 2 pacientes y con anastomosis término-terminal en 1 paciente que requirió pubectomía parcial, con buenos resultados para ambas técnicas. Debe mencionarse que la longitud extensa de la estenosis repercute en los resultados quirúrgicos de ambas técnicas. Algunos autores $^{16}$ reportan estenosis de hasta $16 \mathrm{~cm}$, que no encontramos en esta serie de pacientes. Cuando la estenosis es muy larga pueden ser necesarios dos o más tiempos quirúrgicos, lo que contribuye también a unos resultados desfavorables ${ }^{17}$.

La diferencia en la puntuación del IPSS preoperatoria y posoperatoria de los pacientes operados de uretroplastía con anastomosis término-terminal tuvo un valor $p=0.0011$, y para la anastomosis con injerto de mucosa bucal fue $p=0.00001$ (Tabla 4), lo que indica que ambas técnicas mejoran los síntomas de los pacientes con estenosis uretral anterior ${ }^{10}$.

Antes de la cirugía, la mayoría de los pacientes (93.1\%) presentaron sintomatología urinaria grave y el $6.9 \%$ restante tuvo sintomatología urinaria moderada. En el posoperatorio, ambos grupos tuvieron una mejoría significativa determinada por los resultados de la uroflujometría a los 6 meses del procedimiento. En los pacientes intervenidos con anastomosis término-terminal, la diferencia entre los valores de la uroflujometría preoperatoria y posoperatoria dio un valor $p=0.0046$, y para los operados con anastomosis con injerto de mucosa bucal fue $p=0.00062$ (Tabla 4), lo que indica que los resultados de la uroflujometría mejoran en el posoperatorio a 6 meses con ambas técnicas ${ }^{17-19}$.

Con respecto a las complicaciones, no hubo diferencia significativa entre ambas técnicas, con una $p>0.05$ (prueba exacta de Fisher) 
Tabla 3. Asociación entre las complicaciones posquirúrgicas y el procedimiento realizado

\begin{tabular}{|c|c|c|c|c|c|c|}
\hline & Sin complicaciones & Complicaciones menores & Complicaciones mayores & Total & E de Fisher & $p$ \\
\hline Uretroplastía término-terminal & 5 & 2 & 3 & 10 & 1.74 & NS \\
\hline $\begin{array}{l}\text { Uretroplastía con injerto de } \\
\text { mucosa bucal }\end{array}$ & 12 & 5 & 2 & 19 & & \\
\hline Total & 17 & 7 & 5 & 29 & & \\
\hline
\end{tabular}

Tabla 4. Resultado del IPSS posoperatorio de los pacientes incluidos en el estudio

\begin{tabular}{lcccc}
\hline & \multicolumn{2}{c}{ IPSS posoperatorio } & Total \\
\cline { 2 - 4 } & Leve & Moderado & Grave \\
\hline Operados con uretroplastía término-terminal & 3 & 5 & 2 & 10 \\
Operados con uretroplastía con injerto de mucosa bucal & 10 & 7 & 2 & 19 \\
Total & 13 & 12 & 4 & 29 \\
\hline IPSS: Escala Internacional de Síntomas Prostáticos. & & &
\end{tabular}

IPSS: Escala Internacional de Síntomas Prostáticos.

Tabla 5. Diferencia entre las medias preoperatorias y posoperatorias del IPSS y de la uroflujometría

\begin{tabular}{|c|c|c|c|c|c|c|}
\hline & \multicolumn{6}{|c|}{ Tipo de cirugía } \\
\hline & \multicolumn{3}{|c|}{ Anastomosis término-terminal } & \multicolumn{3}{|c|}{ Injerto de mucosa oral } \\
\hline & Basal & Final & $p$ & Basal & Final & $p$ \\
\hline IPPS & $21.4 \pm 3.6$ & $14.8 \pm 7.96$ & $\leq 0.05$ & $23.5 \pm 2.4$ & $11.73 \pm 5.6$ & $\leq 0.05$ \\
\hline Uroflujometría & $10.08 \pm 0.83$ & $13.83 \pm 3.06$ & $\leq 0.05$ & $13.21 \pm 2.67$ & $10.36 \pm 8.3$ & $\leq 0.05$ \\
\hline
\end{tabular}

IPSS: Escala Internacional de Síntomas Prostáticos.

Una de las limitantes del estudio es que la selección de los pacientes para el tipo de cirugía no fue aleatoria; sin embargo, la comparación de los valores preoperatorios de la longitud de la estenosis uretral, así como de los valores del IPSS y de la uroflujometría, no mostraron diferencias estadísticamente significativas. Otra limitante del estudio es el tamaño de la muestra, por lo que consideramos que estos resultados pueden servir de base para la realización de estudios posteriores con un mayor número de pacientes.

Derivado de los datos presentados, se concluye que ambos procedimientos ofrecen buenos resultados y tienen complicaciones similares, y que los síntomas del tracto urinario mejoran significativamente con las dos técnicas, pero la prueba de efecto entre grupos no muestra diferencia significativa.

\section{Bibliografía}

1. Jordan GH. Cirugía del pene y la uretra. En: Wein AJ, Kavoussi LR, Partin AW, Novick AC, editores. Campbell-Walsh Urología. Buenos Aires: Médica Panamericana; 2007. p. 1023-96.

2. Wong E, Tse V, Wong J. Durability of Memokath ${ }^{\mathrm{TM}}$ urethral stent for stabilisation of recurrent bulbar urethral strictures-medium-term results. BJU Int. 2014;113(Suppl. 2):35-9.

3. Smith TG. Current management of urethral stricture disease. Indian J Urol. 2016;32:27-33

4. Gómez R, Marchetti P, Castillo OA. Rational and selective management of patients with anterior urethral stricture disease. Actas Urol Esp. 2011;35:159-66.

5. Serrano-Brambila EA, Moreno-Alcázar OM, Neri-Páez E, Sánchez-Martínez LC, Hernández-Ordóñez OF, Morales-Morales A. La estenosis de uretra postraumática en el hombre. Rev Med Inst Mex Seguro Soc. 2013;51:472-9.

6. Barbagli G. Adherence to the right diagnostic tools for best outcomes in urethral reconstructive surgery. Eur Urol. 2006;50:424-5.

7. McCallum RW, Colapinto V. The role of urethrography in urethral disease. Part I. Accurate radiological localization of the membranous urethra and distal sphincters in normal male subjects. J Urol. 1979;122:607-11.

8. Cruz García-Villa P, Schroede-Ugalde M, Landa Soler-Martín M, Mendoza-Peña F. Factores de riesgo para el desarrollo de estenosis de uretra en pacientes operados de resección transuretral de próstata. Rev Mex Urol. 2013;73:166-74. 
9. García de León Gómez JM, Vanzzini Guerrero MA, López Chente J. Uretroplastía abierta para corrección de estenosis de uretra anterior. Bo Coleg Mex Urol. 2016;31:14-20.

10. Díaz R, Valdés L. Uretroplastía: experiencia del Servicio de Urología de Hospital Regional de Talca. Rev Chil Urol. 2014;79:66-70.

11. Singh O, Gupta S, Arvind N. Anterior urethral strictures: a brief review of the current surgical treatment. Urol Int. 2011;86:1-10.

12. Droller MJ. Urogenital system - anatomy and histology. En: Droller MJ, editor. Surgical management of urologic disease: an anatomic approach. St Louis: Mosby Year Book; 1992. p. 1218-37.

13. Cisneros-Chávez R, Aragón-Castro M, Morales-Ordaz O. Estenosis uretral: etiología y tratamiento. Experiencia en el Centro Médico ISSEMYM. Rev Mex Urol. 2013;73:229-36.

14. Trujillo Ortiz L, Garza-Sainz G, Mayorga-Gómez E, Osornio-Sánchez V Cornejo-Davila V, Uberetagoyena-Tello I, et al. Uretroplastía término-terminal para el tratamiento primario de la estenosis de uretra: experiencia del Hospital General Dr. Manuel Gea González. Rev Mex Urol. 2016;76:275-8.
15. Mayorga-Gómez E, Gómez SJ, Herrera-Muñoz JA, Preciado-Estrella DA, Sedano-Basilio JE, Trujillo-Ortiz L, et al. Uretroplastía anastomótica de aumento en el manejo de la estenosis de uretra bulbar. Rev Mex Urol. 2015;75:240-3.

16. Roca-Edreira A, Aguilera Tubet C, García-Montesinos Perea BM, Hernández Rodríguez R. Uretroplastía con mucosa oral en estenosis de uretra anterior. Actas Urol Esp. 2008;32:517-21.

17. Lozano-Ortega JL, Pertusa-Peña C. Surgical treatment of urethra stenosis. Results of 100 urethroplasties. Arch Esp Urol. 2009;62: 109-14.

18. Marchal C, Pérez JE, Herrera B, Saez F, Castillo E, Antuña F, et al. Uretroplastía dorsal según técnica de Barbagli. Análisis de resultados y factores de éxito. Arch Esp Urol. 2010;63:537-45.

19. Ramírez-Pérez EA, López-Silvestre JC, Pérez-Elizalde J, Elías-Zonana F. Uretroplastía de mínima invasión con mucosa oral para el manejo de estenosis complejas de uretra anterior en un solo tiempo. Rev Mex Urol. 2012;72:63-71. 